\title{
EFECTO DE LA DOSIS Y FORMULACIÓN DE Metarhizium anisopliae (Mertsch) EN EL COMBATE DE CERCÓPIDOS EN EL PASTO BRACHIARIA (Brachiaria ruzizensis) ${ }^{1}$
}

\author{
Yannery Gómez²
}

\begin{abstract}
RESUMEN
El objetivo de este estudio fue determinar el efecto de diferentes dosis y formulaciones de Metarhizium anisopliae en el combate de cercópidos en el pasto Brachiaria (Brachiaria ruzizensis). Las tres especies de la familia Cercopidae encontradas fueron, Zulia vilior (especie dominante), Aeneolamia lepilior y Aeneolamia reducta. En la dinámica poblacional de estas especies, muchos de los picos generacionales coinciden con los meses de mayor precipitación, por lo que como estrategia de control, se recomienda hacer aplicaciones del hongo en toda el área dañada con las primeras lluvias. Según la fecha de aplicación del hongo $M$. anisopliae, se logra reducir la población de adultos y de ninfas de cercópidos. Para el combate de adultos, se sugiere utilizar la concentración de $1,25 \times 10^{12}$ conidios/ha cuando se aplica por primera vez, con el objeto de inundar el área tratada; posteriormente se recomienda continuar el ciclo de aplicaciones empleando una concentración 6,25 X 1011 conidios/ha haciendo por lo menos, de dos a tres aplicaciones por año. En relación con las formulaciones evaluadas se encontró que el aceite, talco y agua, pueden usarse como vehículos para la aplicación del hongo. Cuando se presentan altas poblaciones de ninfas, se recomienda implementar un Manejo Integrado de la Plaga haciendo uso de manera oportuna de las siguientes herramientas: pastos resistentes, adecuada fertilización, sobrepastoreo cuando hay altas poblaciones de ninfas, una primera aplicación de insecticida en potreros que tengan un historial de altas poblaciones de insectos y continuar con aplicaciones de $M$. anisopliae en las dosis recomendadas.
\end{abstract}

Palabras clave: $M$. anisopliae, pastos, cercópidos, hongos entomopatógenos, $Z$. vilior.

\section{INTRODUCCIÓN}

La familia Cercopidae cuyo estadío ninfal produce un mucílago en la base de los tallos de muchas especies de poáceas y cuya apariencia le otorga el nombre de "baba de culebra" o "salivazo", es sin duda uno de los grupos de insectos más dañinos para los pastos en Costa Rica y en el resto de América Latina. Diferentes especies atacan a las poáceas desde el sur de los Estados Unidos has- ta la Argentina tal y como lo indican Barnard y Frankel (1964), citados por (Skerman y Riveros 1992). En Costa Rica, la distribución de estos insectos es variable ya que, en las zonas altas predominan Prosapia distanti Lall y Prosapia sp. cercana a la bicinta; en regiones bajas y medias se encuentra Zulia vilior costarricensis, mientras que en zonas bajas se presentan Aeneolamia contigua y Prosapia simulans (Vinton y León 2005, en prensa).

1 Parte de tesis de maestría. Programa Posgrado en Protección de Cultivos. Universidad de Costa Rica.

2 Instituto Nacional de Innovación y Transferencia en Tecnología Agropecuaria (INTA). Costa Rica. Correo electrónico: yangomez@ costarricense.cr 
Durante el período de lluvia, la población de estos insectos es muy alta y, debido al daño que producen en sus hojas, los pastos se muestran bronceados y secos, con necrosis similares a las que ocurren en períodos de sequía. Este deterioro lo ocasionan tanto las ninfas como los adultos; sin embargo, los mayores perjuicios se dan con los adultos que se alimentan de las hojas y les inyectan sustancias que intoxican la planta, la cual eventualmente se seca (Hernández 1996).

Metarhizium anisopliae es uno de los hongos entomopatógenos más utilizados y estudiados en el control biológico. Este hongo es un entomopatógeno facultativo capaz de crecer saprofíticamente, pero su asociación con insectos, es probablemente su modo principal de vida. Asimismo, dependiendo de la raza, $M$. anisopliae puede parasitar a un amplio ámbito de insectos plaga y de presentar diversos grados de virulencia hacia los diferentes estadíos del hospedero. Esta variabilidad en la virulencia hace que la selección de aislamientos a utilizar en programas de control de insectos sea importante (Shannon 1994). Para lo anterior, deben producirse grandes cantidades del hongo y mantener su capacidad de infección por un período de tiempo considerable (Carballo 1998).

Una de las desventajas del control de plagas con entomopatógenos, es que éstos necesitan entre otros factores, condiciones favorables de temperatura y luminosidad, para que se tornen epizoóticos (Alves 1986). La enfermedad presenta un carácter epizoótico cuando determinada población de insectos en un período corto de tiempo, muere a causa de un determinado patógeno, presentando niveles con variaciones aleatorias y extremas (Lecuona 1996). El foco primario, (la aplicación del hongo) da como resultado focos secundarios, (individuos infectados); los patógenos, consecuentemente, pueden permanecer en el área no solo en los cadáveres sino, además, como acontece con los virus y protozoarios, pueden pasar de generación en generación a través de los huevos de los insectos (Alves 1986).

Para un control efectivo del salivazo en pastos, es importante determinar la dosis óptima y económicamente aceptable de $M$. anisopliae, y la formulación que permita el establecimiento del hongo en el campo y que se ajuste mejor a las diferentes situaciones geográficas.

Por lo anterior, el objetivo de este trabajo fue determinar el efecto de diferentes dosis y formulaciones de Metarhizium anisopliae, en el combate de cercópidos en el pasto Brachiaria (Brachiaria ruzizensis), lo mismo que identificar y conocer la fluctuación poblacional de las especies de cercópidos presentes en el lugar de estudio.

\section{MATERIALES Y MÉTODOS}

El trabajo de campo se realizó en una finca ganadera, ubicada en Sabanilla de Coto Brus (Puntarenas), región situada en una zona clasificada como tropical húmeda, con uno o dos meses secos; una temperatura máxima es de $29,3^{\circ} \mathrm{C}$, una mínima de $18,7^{\circ} \mathrm{C}$ y una media de $24^{\circ} \mathrm{C}$, con una precipitación mínima anual de $1820 \mathrm{~mm}$ y una precipitación máxima anual 3420 mm (Herrera y Gómez 1993).

Se utilizó un área total de 4,5 ha, dividida en apartos en donde predominó el pasto Brachiaria ruziziensis cv pasto ruzzi. Otras especies presentes en el área de estudio fueron las malezas conocidas como Navajuela (Paspalum virgatum L.), Canutillo (Commelina difusa Burm) y algunas especies pertenecientes a la familia Ciperaceae.

\section{Reproducción de Metarhizium anisopliae}

El aislamiento del hongo utilizado fue obtenido en DIECA, donde se denomina (PL 43 DIECA) y se usa en el combate del salivazo 
en la caña de azúcar. Su reproducción se llevó a cabo en el Laboratorio de Fitoprotección del INTA.

Para asegurar la calidad del material utilizado y revigorizar la cepa, se inocularon larvas de Diatraea sp. con conidios de $M$. anisopliae del aislamiento mencionado. Cuando murieron los insectos y, esporuló el hongo, se aisló nuevamente en cajas de petri con el medio de cultivo PDA (papa-dextrosa-agar).

La metodología para la multiplicación del hongo consistió en colocar 400 gramos de arroz seco (95\% entero) y grano precocido en bolsas de polipropileno. Posteriormente, las bolsas con arroz fueron colocadas en una autoclave a $121{ }^{\circ} \mathrm{C}$ y una atmósfera de presión durante 20 minutos. Para la inoculación, se adicionaron $15 \mathrm{ml}$ por bolsa de la suspensión del hongo preparado a partir de los platos con hongo esporulado, a una concentración aproximada de $1 \times 10^{10}$ conidias $/ \mathrm{ml}$.

Una vez que el micelio colonizó la superficie del grano (de cuatro a seis días después de la inoculación), el material se colocó en bandejas plásticas de $25 \mathrm{~cm}$ x $30 \mathrm{~cm}$ y $5 \mathrm{~cm}$ de altura, donde se mantuvo 12 días con el objeto de favorecer su esporulación. Finalmente, se removió parcialmente la tapa para secar el producto y obtener la concentración adecuada.

Los conidios se separaron del arroz con un vibrador con tamiz de 40 huecos por pulgada lineal (MESH 40), luego se cuantificó su concentración por gramo y se estimó la cantidad de producto a utilizar, según la concentración.

\section{Identificación de especies de cercópidos y fluctuación poblacional}

Para identificar y conocer la fluctuación poblacional de estos cercópidos en el tiempo, se colectaron adultos a partir de julio de 2000 hasta julio 2001 y se graficó la suma total por especie encontrada mensualmente. Con los adultos, se utilizó una red entomológica estándar, dando tres pases de red en cada par- cela de $100 \mathrm{~m}^{2}$ caminando en zigzag, para un total de área de $3.600 \mathrm{~m}^{2}$. Con las ninfas (Figura 1) se usó un marco de $35,5 \mathrm{~cm}$ de lado, el cual se lanzó una vez por parcela de $100 \mathrm{~m}^{2}$ al azar, para un total de área de 3.600 $\mathrm{m}^{2}$. No se hizo distinción por especie, ya que en campo es muy difícil separarlas; los insectos colectados se llevaron al laboratorio para su identificación.

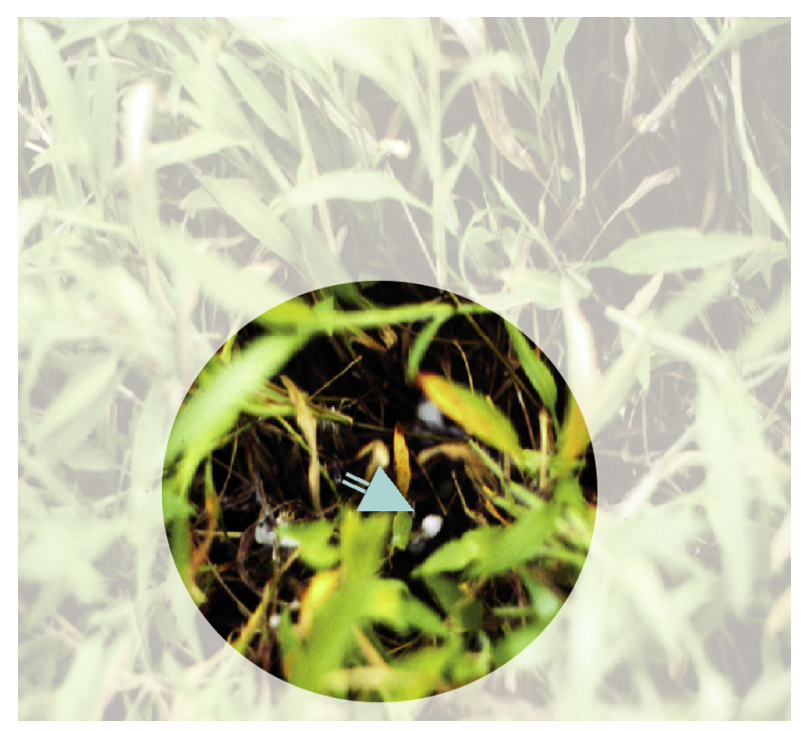

Figura 1. Ninfas recubiertas por el mucílago que producen, del cual se deriva el nombre de "salivazo".

\section{Eficacia de tres dosis de M. anisopliae for- muladas en agua, aceite-agua y talco}

El ensayo se dispuso en un diseño de bloques completos al azar con cuatro repeticiones, con una parcela experimental de $10 \mathrm{mx}$ $10 \mathrm{~m}$, y una parcela útil de $2 \mathrm{~m}^{2}$. Se evaluaron tres concentraciones del hongo Metarhizium anisopliae: 2,5 X $10^{12}$ conidios/ha; 1,25 $X 10^{12}$ conidios/ha y $6,25 \times 10^{11}$ conidios/ha (Cuadro 1). Las aplicaciones del hongo se realizaron en la tarde (3 p.m. en adelante) o, muy temprano en la mañana (5-7 a.m.).

Las conidias fueron preparadas en tres formulaciones: Agua + conidias, aplicada con bomba de motor para favorecer la homogeneidad de la suspensión, se agregaron cinco 
cc/bomba del coadyuvante NP7, a razón de cinco cc por bomba; Talco + conidias, aplicada al voleo y mezclada con $400 \mathrm{~g}$ de talco inerte / parcela; y Aceite-agua + conidias, aplicada con bomba de motor; se agregaron cinco cc/bomba de coadyuvante NP7. Además se adicionó aceite agrícola en una concentración del 20\%. El Cuadro 1, presenta los tratamientos utilizados.

Cuadro 1. Detalle de los tratamientos con las diferentes dosis y formulaciones. Coto Brus, 2000 - 2001

\begin{tabular}{|c|c|c|c|}
\hline \multirow[t]{2}{*}{ Formulación } & \multicolumn{3}{|c|}{ Concentración } \\
\hline & $2,5 \times 10^{12}$ & $1,25 \times 10^{12}$ & $6,25 \times 10^{11}$ \\
\hline Agua & $x$ & & \\
\hline Agua & & $\mathrm{x}$ & \\
\hline Agua & & & $\mathrm{x}$ \\
\hline Aceite-agua & $\mathrm{x}$ & & \\
\hline Aceite-agua & & $\mathrm{x}$ & \\
\hline Aceite-agua & & & $\mathrm{x}$ \\
\hline Talco & $x$ & & \\
\hline Talco & & $x$ & \\
\hline Talco & & & $\mathrm{x}$ \\
\hline Testigo & & & \\
\hline
\end{tabular}

El hongo se aplicó en todas las parcelas una vez por mes en agosto, setiembre y octubre del año 2000 y en mayo, junio y julio del año 2001.

Todos los adultos de cercópidos colectados en la parcela se llevaron al laboratorio para su identificación. Los adultos vivos se conservaron en alcohol y los muertos, con sospechas o con síntomas de infección, se llevaron en bolsas plásticas dentro de hieleras, para corroborar a nivel de laboratorio si efectivamente fueron infectados por $M$. anisopliae.

Las variables evaluadas fueron: a- densidad de adultos y ninfas de cercópidos antes y después de la aplicación; y b- número de adultos muertos (cinco-ocho días después de la aplicación).
Se realizó un análisis de varianza entre los tratamientos con las diferentes formulaciones y las concentraciones evaluadas, para lograr un mayor ajuste a la curva normal los datos fueron transformados a $\sqrt{ }(x+0,5)$. Se practicaron análisis de varianza con el paquete estadístico SAS y se compararon los promedios de los adultos y ninfas capturados con la prueba de Duncan.

\section{RESULTADOS}

\section{Identificación de especies de cercópidos y fluctuación poblacional}

Se encontraron e identificaron tres especies de la familia Cercopidae: Zulia vilior (Figura 2), que fue la especie dominante; $A e-$ neolamia lepilior, segunda especie en importancia y finalmente Aeneolamia reducta, encontrada ocasionalmente, según la clave utilizada por Vinton y León (2005).

La Figura 3 presenta la precipitación mensual, la fluctuación poblacional, los momentos de aplicación del hongo (identificados con flechas rojas) y el conteo total de ninfas.

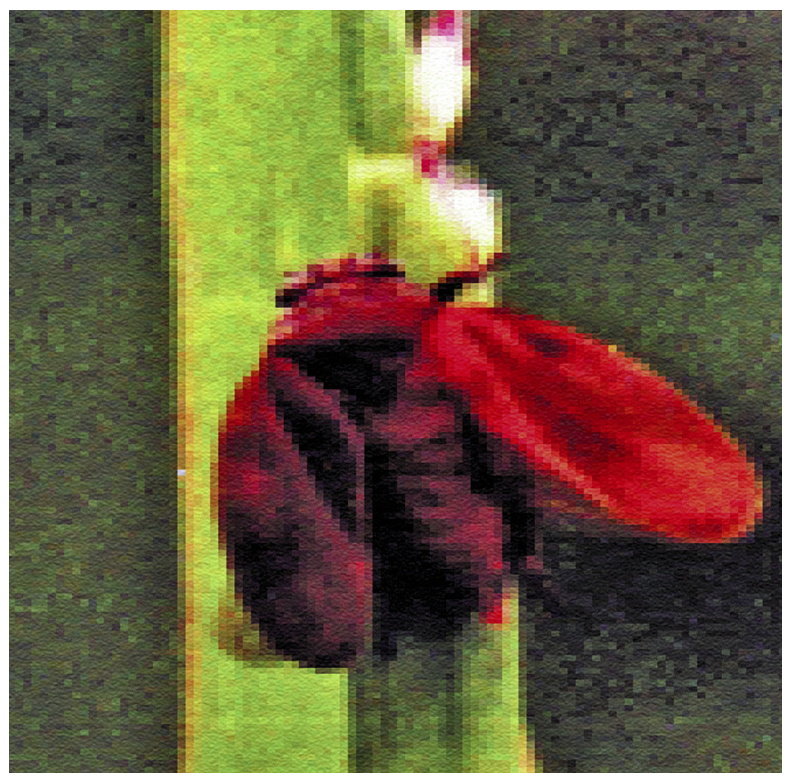

Figura 2. Adulto de Zulia vilior especie dominante en este estudio. 


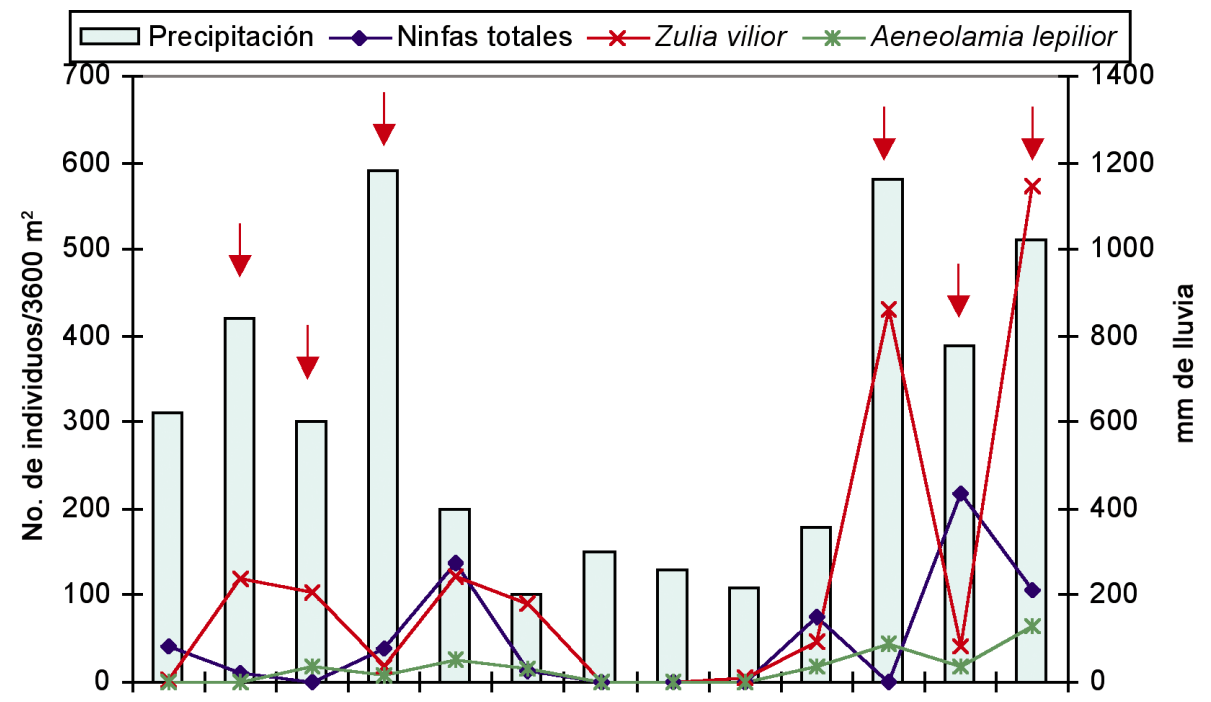

Figura 3. Precipitación mensual y fluctuación poblacional de cercópidos en pasto Brachiaria ruziziensis. Sabanilla de Coto Brus, Puntarenas. Período 2000 - 2001.

La misma figura indica que en el año 2000 ocurrieron dos picos importantes de población los cuales coincidieron con los meses lluviosos. Entre agosto y septiembre se capturaron 225 individuos de Zulia vilior, mientras que entre noviembre y diciembre, se capturaron 213 individuos. En estos períodos se realizaron aplicaciones del hongo para bajar las poblaciones. De diciembre y hasta marzo (estación seca) se presentó una notable disminución en la población de este insecto, situación que se debe a que los huevos colocados en el suelo, no eclosionan, sino que entran en lo que se conoce como estado de diapausa, el cual se rompe hasta que ocurren las primeras lluvias (Fontes et al. 1995).

Al caer las primeras lluvias en abril del 2001, de los huevos que se encuentran en diapausa, nacen nuevas ninfas por lo que la población empieza a crecer generando un nuevo pico poblacional del año, en este momento se realizó la primera aplicación del entomopatógeno. En el mes de julio ocurrió un nuevo pico poblacional y se aplicó nuevamente el hongo $M$. anisopliae. En ese mismo mes, la especie que aumentó de manera importante la población de adultos fue Zulia vilior.
El comportamiento en la fluctuación poblacional de $A$. lepilior coincidió con el de $Z$. vilior. En los meses de agosto y septiembre, noviembre y diciembre, aumentó su población, con una captura promedio de 40 individuos. Nuevamente, en mayo del 2001 se presentó un fuerte crecimiento en la población, como resultado de las fuertes lluvias del mes anterior.

De la especie $A$. reducta se encontró un individuo en septiembre de 2000, y otro en mayo de 2001.

Las poblaciones de ninfas fueron bajas en los meses de agosto y setiembre del 2000, posiblemente como consecuencia del "Fenómeno de la Niña", el cual de acuerdo con el criterio de los productores de la zona, ocasionó lluvias de menor intensidad en comparación con otros años. Se da un nuevo aumento de población de ninfas para junio del 2001 (Figura 3).

Con las primeras lluvias en abril del 2001, subió el nivel de población de ninfas a 125 individuos. Para el mes de junio del mismo año se dio el pico más alto de población donde se contó un total de 438 ninfas. 


\section{Eficacia de tres dosis de $M$. anisopliae y formulaciones: agua, aceite-agua y talco}

Para la variable adultos, hubo diferencias significativas en la captura de adultos $(\mathrm{P}<$ 0,0001). Al realizar el contraste del testigo / las parcelas aplicadas con el hongo, el resultado fue altamente significativo $(P<0,0001)$, indicando que al aplicar el hongo $M$. anisopliae se logró bajar las poblaciones de adultos de cercópidos.

No se dieron diferencias de captura entre las dosis y las formulaciones evaluadas, ya que el efecto de los tratamientos fue muy parecido. La fluctuación de los adultos con las diferentes formulaciones de la concentración baja 6,25 X $10^{11}$ con respecto al testigo se muestra en la Figura 4, en la cual se representan con flechas los momentos de aplicación del hongo.

El primer aumento en la población de adultos con un promedio de cuatro adultos/parcela, se dio en septiembre del año 2000, cuando las condiciones climáticas fueron, precipitación $(300 \mathrm{~mm})$, temperatura $\left(26^{\circ} \mathrm{C}\right)$ y humedad relativa que se mantuvo constante en 96\% (Figura 6). En ese momento se realizó la primera aplicación de los tratamientos del hongo.
El segundo pico de captura de adultos fue en noviembre del mismo año, favorecido por las altas precipitaciones y temperaturas del mes anterior, con un promedio de captura de siete adultos/parcela; por lo que se realizó la segunda aplicación del hongo. Por último, el pico de mayor captura de adultos con un promedio 22 adultos/parcela fue en mayo 2001, durante el cual se presentaron las primeras lluvias fuertes y muchas ninfas salieron de su diapausa, por lo que se decidió hacer la primera aplicación de ese año (Figura 4).

En todas las parcelas donde se aplicó hongo hubo menor población de adultos de cercópidos que con el testigo.

Para la variable ninfas, no hubo diferencias entre el testigo y los tratamientos; se presentaron diferencias significativas $(\mathrm{P}<$ $0,0001)$ en las fechas de aplicación. Esto significa que se logró bajar las poblaciones de ninfas con la aplicación de los tratamientos aún cuando se produjo una alta precipitación, esto porque el agua de la lluvia ayudó a bajar la conidia del hongo hasta la base de las cepas donde se encontraba la ninfa, entrando en contacto directo con ella, infectándola y bajando la población.

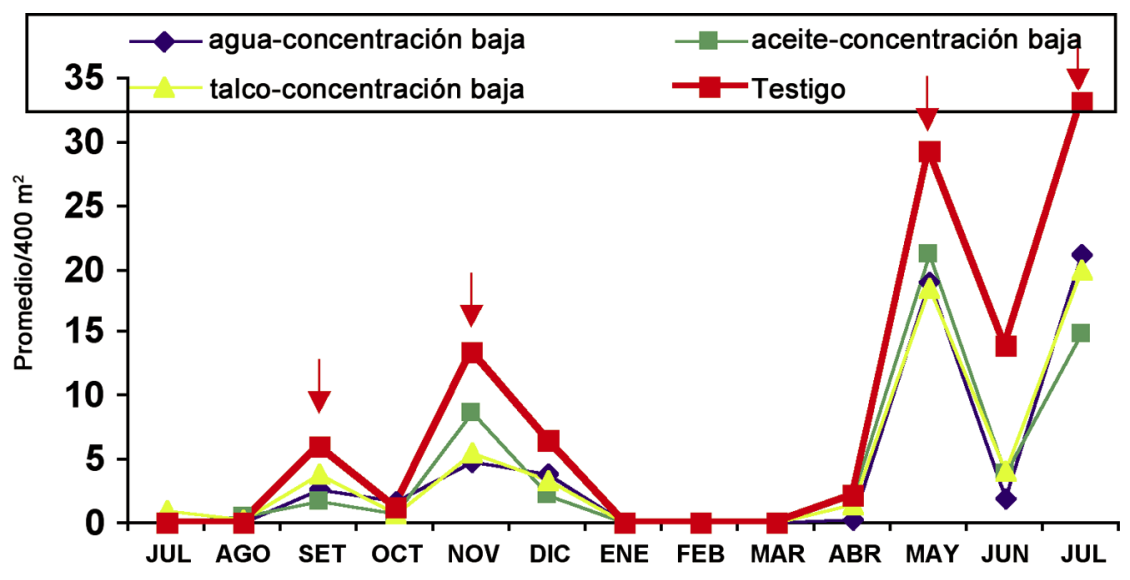

Figura 4. Número de adultos de cercópidos, bajo diferentes formulaciones agua, aceite y talco en la concentración de 6,25 x 1011 conidia/ml de M. anisopliae. Coto Brus. 2000-2001. 
Se dieron diferencias en la colecta de adultos parasitados, con los tratamientos en las diferentes fechas de evaluación. Se encontraron adultos muertos a los ochos días y al mes después de la aplicación en mayor cantidad con las formulaciones talco y agua (Figura 5).

Los momentos donde se encontró el mayor número de adultos parasitados fueron: octubre - diciembre del año 2000 y mayo-julio del 2001. No así en el testigo, donde no se encontraron adultos parasitados

En la Figura 6 se muestra la fluctuación de precipitación, humedad relativa y temperaturas del periodo en el que se realizó el estudio, la cual se tuvo como referencia para comparar la influencia de las lluvias en el aumento de población de los cercópidos.

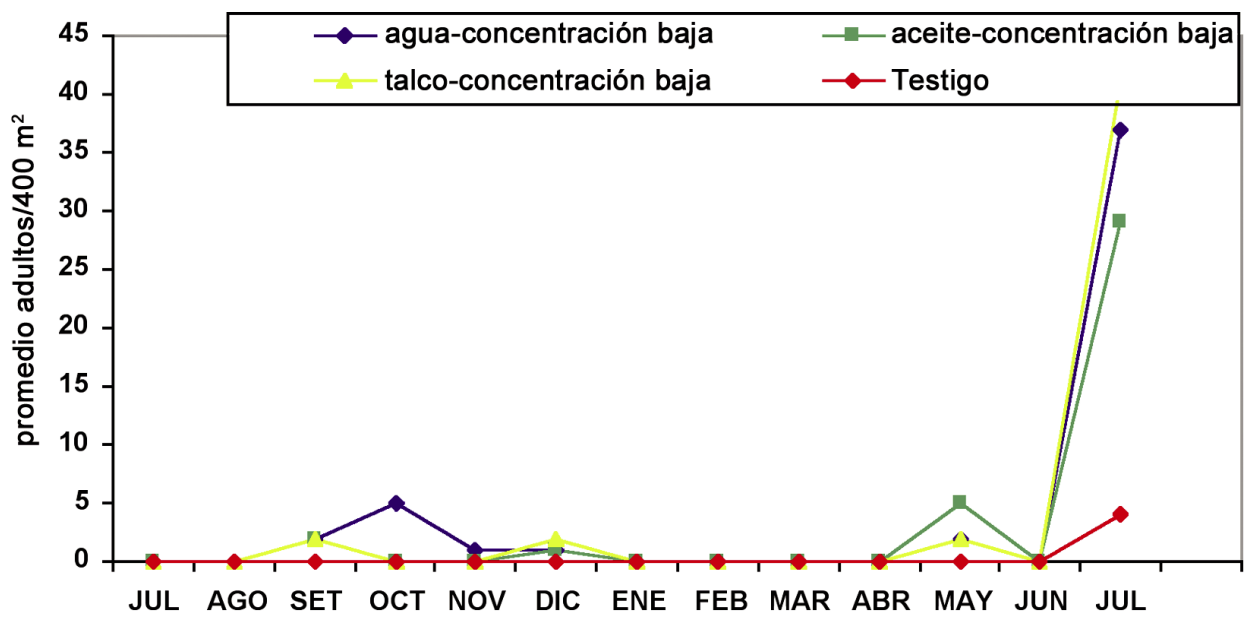

Figura 5. Número de insectos de cercópidos infectados con $M$. anisopliae en concentraciones de $6,25 \times 10^{11}$ conidias $/ \mathrm{ml}$, en tres formulaciones agua, aceite y talco. Coto Brus. 2000-2001.

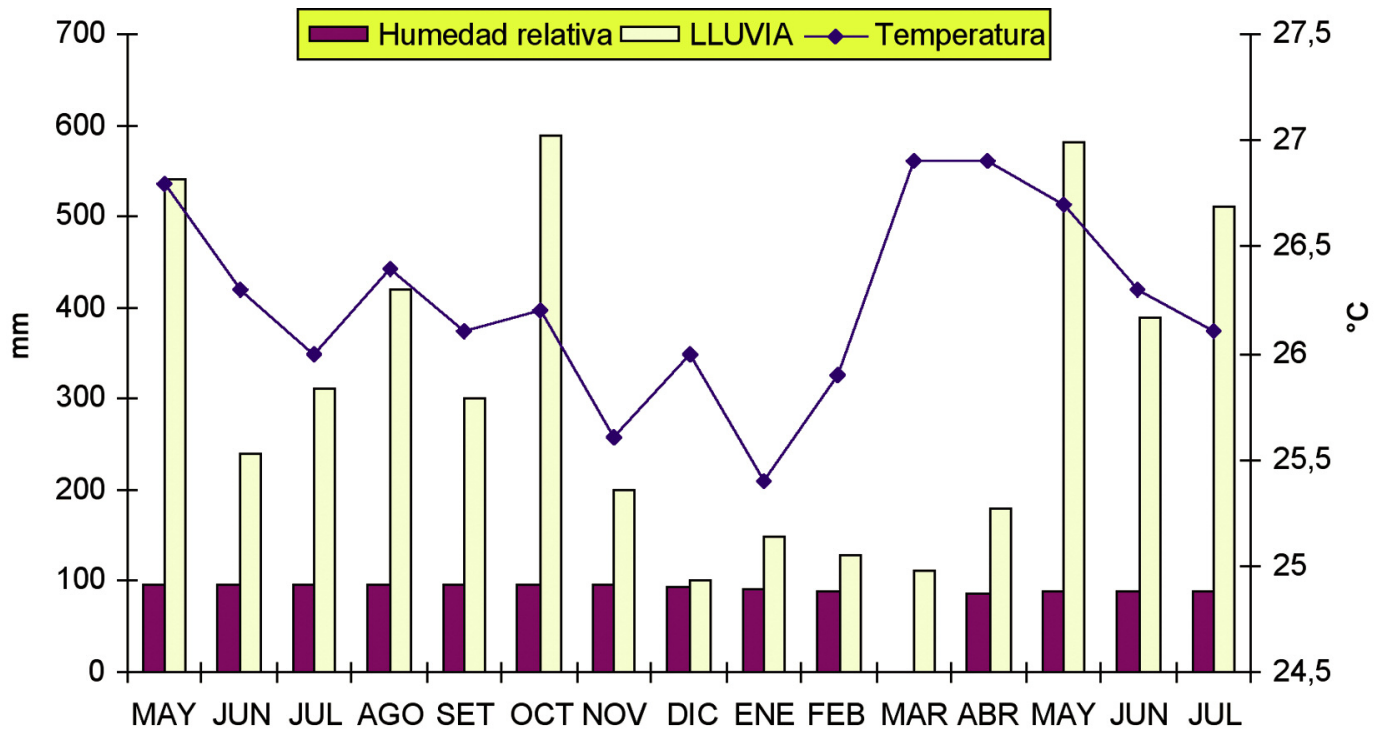

Figura 6. Porcentaje de humedad relativa, lluvia $(\mathrm{mm})$ y temperatura $\left({ }^{\circ} \mathrm{C}\right)$, durante mayo 2000 a julio 2001. Fuente: Instituto Meteorológico Nacional. Coto 47. 
El análisis de varianza realizado entre las formulaciones de agua, de aceite y de talco no presentó diferencias significativas en ninfas y adultos parasitados, aunque hubo diferencias entre las fechas que se aplicó el hongo.

En el caso de las concentraciones tampoco se dieron diferencias significativas entre ellas.

\section{DISCUSIÓN}

\section{Identificación de especies de cercópidos y fluctuación poblacional}

Zulia vilior es una de las especies reconocidas como especie dominante que causa daño en las zonas bajas y medias en nuestro país tanto en el cultivo de caña de azúcar como en pastos, según lo reportado por Vinton y León (2005, en prensa). Se observó que con el aumento en las lluvias, las poblaciones de esta especie se incrementaron. Al aplicar el hongo por primera vez se logró disminuir las poblaciones, sin embargo en la segunda aplicación de $M$. anisopliae la reducción de las poblaciones fue mayor, observándose una acción efectiva del hongo, aún cuando la precipitación fue más alta, comparada con la del mes anterior. Esta alta precipitación permitió nuevamente que las poblaciones del insecto se incrementaran hacia el mes siguiente, haciendo necesario realizar otra aplicación del hongo. Según indica Cisneros (1995), las fluctuaciones están claramente asociadas con las estaciones del año, aunque la mecánica de esta asociación por lo general, no está bien determinada. El incremento y disminución poblacional parecen estar asociados con los factores físicos del ambiente y con la fenología de las plantas hospederas que determinan la relativa disponibilidad de alimentos para la plaga.

Con respecto a la población de ninfas, se observó que al igual como sucedió con los adultos, las altas precipitaciones favorecieron su proliferación y su paso a la fase adulta. Además, en los meses donde hubo más adul- tos, se encontraron consecuentemente al mes siguiente, gran cantidad de ninfas y viceversa. No fue posible distinguir las especies en el estado de ninfa, aunque posiblemente correspondieron en gran cantidad a $Z$. vilior, dado que esta especie predominó en la fase adulta. En la mayoría de los casos, las ninfas se encontraron en la base de las plantas hospederas, pues prefieren lugares sombríos y con alta humedad (Miñón y Pérez 1985).

\section{Eficacia de tres dosis de M. anisopliae y tres formulaciones: agua, aceite-agua $y$ talco.}

Los resultados dejan ver que el entomopatógeno $M$. anisopliae redujo las poblaciones de los adultos de cercópidos, demostrándose que este hongo es efectivo y puede usarse en programas de combate contra el "salivazo". Durante los meses de evaluación hubo variaciones de precipitación y temperatura que favorecieron la población de adultos y ninfas, lo que indica que es necesario realizar varias aplicaciones del hongo durante el año para combatir eficientemente la plaga.

En el caso de las ninfas no se demostró un efecto claro del hongo sobre ellas, ya que en el análisis de variación no se presentaron diferencias de población con respecto al testigo. Sin embargo, los resultados muestran que en las diferentes fechas de la aplicación de $M$. anisopliae se logró reducir las poblaciones de ninfas. Hay varias razones, por ejemplo, por efecto de lluvia, salpique de agua o viento, el hongo baja hasta nivel del suelo, y las ninfas no estuvieron expuestas a la acción directa del hongo. Otra posible razón fue, la misma estructura de la cepa del pasto que era muy densa, formando una especie de colchón protector de la ninfa.

Lecuona y Alves (1996) indican que para poder obtener buenos resultados en el control de ninfas hay que utilizar dosis muy altas del hongo; la dosis mínima que ellos recomiendan es de $5 \times 10^{12}$ conidias/ha y debe aplicarse en el momento de mayor abundancia de ninfas, en nuestro caso las dosis utilizadas fueron 
más bajas y de ahí que no se tuvo un buen efecto de combate contra ellas. En los adultos parasitados, se observaron los siguientes síntomas: se ubicaron en la parte superior de las hojas y abrieron sus alas, perdieron movilidad o se hicieron más lentos, al morir quedaron momificados. Se logró comprobar que depredadores como las hormigas aprovecharon estas circunstancias, atacando a los cercópidos enfermos o muertos, y en varias ocasiones se encontró solo la cabeza.

En el campo, con los cercópidos adultos infectados se pudo comprobar que el hongo invadió al insecto. Se observó micelio entre el cuerpo del insecto y la hoja de pasto; se produjeron conidios nuevos o esporas que podrían diseminarse por acción del viento, agua, el hombre o de otros organismos como lo menciona Lecuona (1996).

Otro aspecto importante que se determinó fue la gran cantidad de adultos parasitados al mes siguiente de la evaluación. Ésto es parte de las ventajas que se señalan de los controladores naturales, que pueden darse reinfecciones en el tiempo, si las condiciones ambientales lo permiten. Lecuona (1996) indica que las oscilaciones presentadas debidas a partir del inóculo secundario que se genera a partir del cuerpo de insectos parasitados; el número o porcentaje de insectos parasitados por un patógeno está en función del tiempo.

Según los resultados, cualquiera de las formulaciones evaluadas, se puede utilizar para aplicar el hongo ya que todas sirven como vehículos para diseminarlo; sin embargo, el agua le proporciona al hongo una condición idónea de humedad necesaria para su estabilidad, el aceite tiene la ventaja que preserva la conidia y en el caso el talco aún cuando resultó ser un buen vehículo para la aplicación, la humedad relativa que necesita el hongo para su existencia debe conseguirla del medio, si no se encuentra, las conidias no germinarán. La formulación en agua es el elemento más disponible y por lo tanto la más utilizada. También se encontró que las tres concentraciones empleadas combaten igualmente al salivazo.

Gómez-Bonilla (2001) observó que la gota de aceite que envuelve la conidia, hace que su germinación sea más lenta, lo cual podría ser beneficioso, si no está expuesta a los rayos solares o a altas temperaturas.

Por supuesto que una de las pretensiones de utilizar control biológico es que el organismo se establezca, pero no debería ser algo condicionante, es una ventaja al usar este tipo de controladores, que el microorganismo se establezca y sea endémico en el campo. El efecto logrado es semejante al de un insecticida químico, de manera que esta opción corresponde al empleo de insecticidas microbianos o bio-insecticidas, además la no contaminación al ambiente, inocuidad para los animales que se alimentan de este tipo de pastos y menores costos comparativos. Se puede empezar con la concentración de 1,25 $X 10^{12}$ conidios/ha y después se puede continuar con la concentración de $6,25 \times 10^{11}$ conidios/ha, para que los costos no sean muy altos. Lecuona y Alves (1996) en sus investigaciones demostraron altos porcentajes de control del salivazo con $M$. anisopliae, con valores en un rango de 10 y hasta $60 \%$ menos de mortalidad.

\section{RECOMENDACIONES}

Se obtuvo un buen combate de los adultos de cercópidos con el uso del hongo entomopatógeno; por lo tanto, para mejorar el combate de ninfas en especies de pasto susceptibles $y$ en fincas con un historial de fuertes ataques de la plaga, se puede recomendar lo siguiente para futuros trabajos: a- conocer la dinámica poblacional de la plaga in situ, bestablecer los momentos de mayor abundancia de ninfas y c- realizar aplicaciones del hongo con dosis altas para el combate de ninfas, preferiblemente con bomba de motor, para ayudar a penetrar más en el pasto. 
Llevar un manejo integrado en potreros que tengan un historial de altas poblaciones de insectos y empezar su combate utilizando pastos resistentes. Se pueden reducir las poblaciones de adultos en la primera generación al aplicar algún insecticida selectivo y pastorear de manera rotativa para reducir las poblaciones de ninfas y aplicar $M$. anisopliae sobre la segunda y tercera generación y continuar en el tiempo con aplicaciones de $M$. anisopliae.

\section{CONCLUSIONES}

1. Se determinó que las especies de la familia Cercopidae encontradas en esa zona fueron, Zulia vilior (especie dominante), Aeneolamia lepilior y Aeneolamia reducta.

2. Se dio variación de la población de estas especies y muchos de los picos coinciden con los meses de mayor precipitación.

3. Cuando se tiene historia de esta plaga en la finca, se deben realizar las aplicaciones del hongo en toda el área de pasto dañada con las primeras lluvias, como estrategia de control. La precipitación y la intensidad y dirección del viento, son factores a considerar en el momento de la aplicación.

4. El hongo M. anisopliae baja la población de adultos y ninfas de cercópidos, según la fecha cuando se aplique, ya que la lluvia o el salpique de agua y el viento, bajan el hongo hasta nivel del suelo.

5. Para el combate de adultos de cercópidos en el cultivo de pasto, se sugiere utilizar la concentración de 1,25 X $10^{12}$ conidios/ha cuando se aplica por primera vez, para que sea de manera inundativa y continuar con la concentración de $6,25 \times 10^{11}$ conidios/ha haciendo por lo menos de dos a tres aplicaciones por año.

6. Las formulaciones aceite, talco y agua pueden ocuparse como vehículos de apli- cación del hongo, aunque el agua es la manera tradicional y la más utilizada. El uso de cualquiera de estas formulaciones estará condicionado por el costo y acceso que se tenga.

7. Un manejo integrado es la mejor manera de combate de este insecto, con pastos resistentes, adecuada fertilización, sobrepastoreo cuando hay altas poblaciones de ninfas, una primera aplicación de insecticida (en potreros que tengan un historial de altas poblaciones de insectos) y continuar con aplicaciones de M. anisopliae.

\section{LITERATURA CITADA}

Alves, S. B. 1986. Controle microbiano de insetos. Brasil, Editora Manole. 277p.

Carvallo V., M. 1998. Formulaciones de hongos entomopatógenos. Revista Manejo Integrado de Plagas (47): i-iv.

Cisneros V., F. 1995. Control de plagas agrícolas. 2 edición. Full Print. Lima, Perú. 313 p.

Fontes, E.G; Pires, C.; Sujii. E. 1995. Mixed riskspreading strategies and the population dynamics of Braziliam. Pastura Pest, Deois flavopicta (Homoptera: Cercopidade). J. Econ. Entomol. 88(5):1256-1262.

Gómez-Bonilla, Y. 2001. Viabilidad y germinación de conidias de Metarhizium anisopliae utilizando tres vehículos de dispersión. Capitulo IV, Tesis de maestría en Protección de Cultivos, Universidad de Costa Rica. 12 p. (En prensa).

Hernández, J. 1992. Algunas estrategias para combatir la "baba de culebra" Coopemontecillos. Revista Informativa del Comité de Educación Bienestar Social (64): 5.

Hernández, J. 1996. Combate de la plaga de la "baba de culebra". Montecillos Boletín oficial. Coopemontecillos, Producción Agrícola. Editorial Desarrollos Gráficos (90): 8.

Herrera S., W.; Gómez P., L.D. 1993. Mapa de unidades bióticas de Costa Rica. Escala 1:685 000 // Color. 
Lecuona, R.; Alves, S 1996. Epizootiología. In: Microorganismos Patógenos empleados en el Control Microbiano de Insectos Plaga. Talleres Gráficos Mariano. Buenos Aires, Argentina. p.17-34.

Lecuona, R.; Alves, S 1996. Utilización de hongos Entomopatógenos. In: Microorganismos Patógenos empleados en el Control Microbiano de Insectos Plaga. Talleres Gráficos Mariano. Buenos Aires, Argentina. p. 241-254.

Miñon, D.; H. Pérez. 1985. Chicharrita de los pastos plaga de las pasturas. Revista del Instituto Nacional de Tecnología Agropecuaria (INTA). Santiago del Estero. 12p.
Skerman, P.J.; Riveros, F. 1992. Gramíneas tropicales. FAO, Organización de las Naciones Unidas para la agricultura y la alimentación. Roma FAO. p 3-8.

Shannon, P. 1994. In: Biología y control de PhyIlophaga spp. Ed por P. Shannon y M. CarbaIlo, 1996. CATIE. Serie Técnica. Informe Técnico No. 277. Turrialba, Costa Rica. p. 80-93.

Vinton, T.; León, R. 2005. La identificación de las especies de salivazo, encontradas en caña de azúcar y pastos (Homoptera:Cercopidae) en Costa Rica. In: Manejo Integrado de plagas y Agroecología. 14 p. (En prensa). 